\title{
Morphological Characteristics of Terminalia of the Wasp-Mimicking Fly, Stomorhina discolor (Fabricius)
}

\author{
Kittikhun Moophayak ${ }^{1}$, Sangob Sanit ${ }^{2}$, Tarinee Chaiwong ${ }^{3}$, Kom Sukontason ${ }^{2}$, \\ Hiromu Kurahashi ${ }^{4}$, Kabkaew L. Sukontason ${ }^{2}$, Roy C. Vogtsberger ${ }^{5}$ and \\ Nophawan Bunchu ${ }^{6,7, *}$
}

1 Nakhonsawan Campus, Mahidol University, 402/1 Moo 5, Khaothong Subdistrict, Phayuhakiri District, Nakhonsawan 60130, Thailand; khun_khithop@hotmail.com

2 Department of Parasitology, Faculty of Medicine, Chiang Mai University, 110 Inthavaroros Road, Muang District, Chiang Mai 50200, Thailand; sang.ngeab@gmail.com (S.S.); kom.s@cmu.ac.th (K.S.); kabkaew.s@cmu.ac.th (K.L.S.)

3 College of Medicine and Public Health, Ubon Ratchathani University, 85 Sathonlamark Road, Warin Chamrap District, Ubon Ratchathani 34190, Thailand; trainee.4004@gmail.com

4 Department of Medical Entomology, National Institute of Infectious Diseases, Tokyo 162-8640, Japan; mlb15110@nifty.com

5 Department of Biology, Midwestern State University, 3410 Taft Boulevard, Wichita Falls, TX 76308, USA; roy.vogtsberger@mwsu.edu

6 Department of Microbiology and Parasitology, Faculty of Medical Science, Naresuan University, 99 Moo 9, Tha Pho Subdistrict, Muang District, Phitsanulok 65000, Thailand

7 Centre of Excellence in Medical Biotechnology, Faculty of Medical Science, Naresuan University, 99 Moo 9, Tha Pho Subdistrict, Muang District, Phitsanulok 65000, Thailand

* Correspondence: bunchu_n@hotmail.com; Tel.: +66-5-596-4716

Academic Editor: Brian T. Forschler

Received: 2 December 2016; Accepted: 9 January 2017; Published: 12 January 2017

\begin{abstract}
Stomorhina discolor (Fabricius), a species of blow fly that mimics wasps, is distributed worldwide, but detailed information about characteristics of its adult terminalia is incomplete. To help fill this gap in the information, the morphology of adult stages of $S$. discolor was investigated using light microscopy and scanning electron microscopy. Observations using the light microscope revealed unique characteristics of the male genitalia that are markedly different from other blow fly species. More morphological detail, including observation of several sensilla (e.g., sensilla trichoid and sensilla basiconica) along the male terminalia and female ovipositor, was seen under the scanning electron microscope. These details can be taxonomically valuable for identifying males and females of $S$. discolor and may help address matters concerning copulation in this species.
\end{abstract}

Keywords: Stomorhina discolor; ultrastructure; external genitalia; ovipositors

\section{Introduction}

Stomorhina discolor (Fabricius, 1794) is a blow fly species in the subfamily Rhiniinae and is known as the wasp-mimicking fly. It has been recorded in many parts of the world [1-5]. Biological information for this species is limited. In Thailand, research in Phitsanulok Province of the lower northern region of the country revealed that this species was the most commonly collected blow fly species of the subfamily Rhiniinae, and it can be found in several types of areas including agricultural, mountainous, and forested areas [6]. Senior-White et al. [4] noted that larvae of this species were found in nests of the ant Camponotus angusticollis (Jerdon), among the roots of Hevea brasiliensis Muell. Arg. affected by the fungus Botryodiplodia theobromae Patton, and riddled with small boring beetles. Adults are occasionally attracted by opening an ant nest. Males are known to often exhibit a hovering behavior 
in small swarms under trees [4]. Among the morphological descriptions of S. discolor that have been published, only limited terminalia illustrations of male genitalia of the species have been provided [4]. Molecular phylogeny of this species has been analyzed by Kutty et al. [7].

In order to elaborate the biological information of this fly species, morphological features of both male and female terminalia of $S$. discolor were studied by using light microscopy (LM) and scanning electron microscopy (SEM).

\section{Materials and Methods}

\subsection{Wasp-Mimicking Fly, S. discolor}

In December 2012, we discovered numerous blow fly specimens of $S$. discolor near a blooming Buchanania lanzan Spreng tree at Mahidol University Nakhonsawan Campus, Nakhonsawan Province in the north-central part of Thailand $\left(15.58189^{\circ} \mathrm{N}, 100.14640^{\circ} \mathrm{E}\right)$. Individual flies were observed moving over the flowers and were collected using a sweep net. They were anesthetized in bottles with ethyl acetate solution and transported back to the lab for species confirmation under a stereomicroscope (Olympus, Tokyo, Japan) using the taxonomic key of Kurahashi and Bunchu [2].

As seen in Figure 1, members of $S$. discolor are small-medium size blow flies with a dark colored thorax with dense pollinosity. Wings are transparent with an apical dark spot in both sexes. The abdomen is black or brown posteriorly with a contrasting yellow on tergites $1+2$. Males are easily distinguished from females by their holoptic eyes; whereas, the eyes of females are dichoptic. After specific identity of specimens had been confirmed, male and female flies of $S$. discolor were individually selected to further investigate the ultrastructural morphology of their external genitalia.
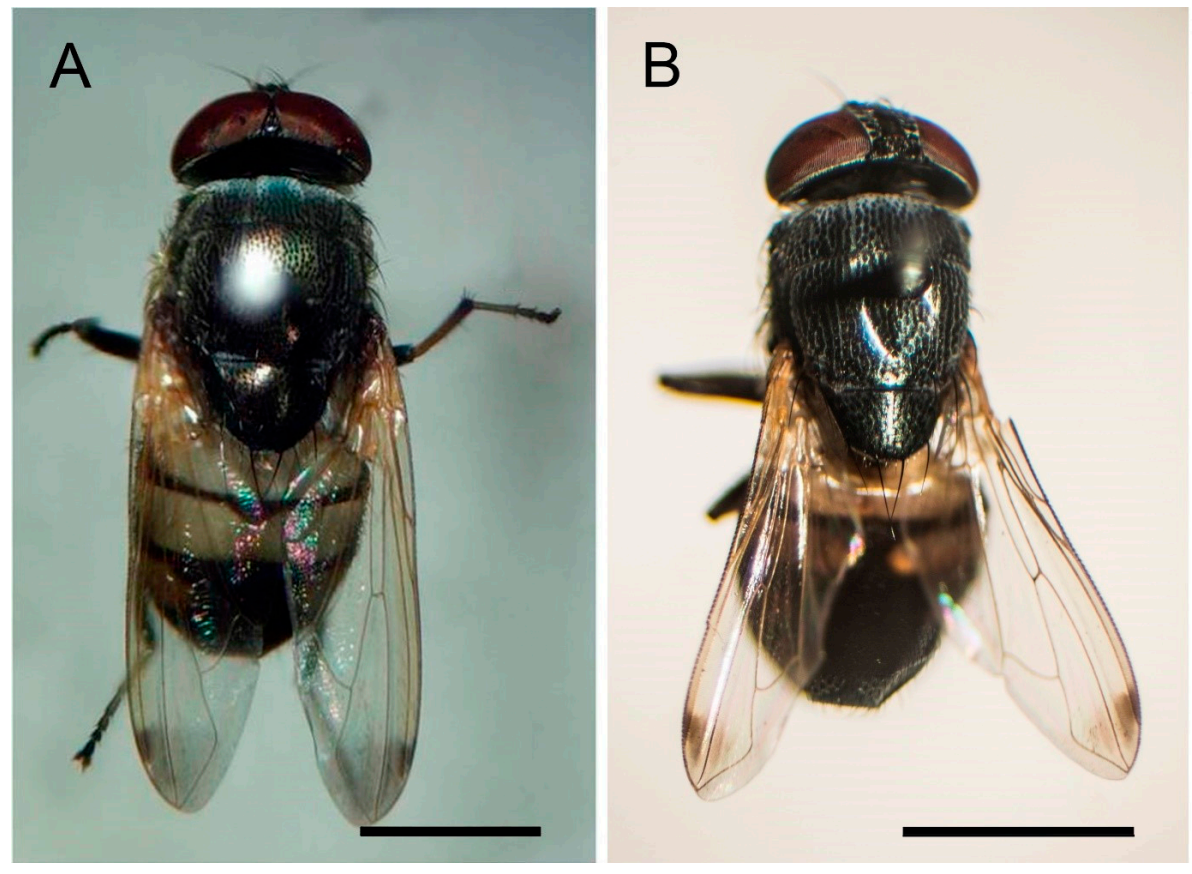

Figure 1. Dorsal view of Stomorhina discolor. (A) Male with holoptic eyes; (B) Female with dichoptic eyes $($ bar $=2 \mathrm{~mm})$.

\subsection{Light Microscopy}

Fifteen fly specimens of each sex were cut between tergites 4 and 5 using a sharp forceps under a stereomicroscope to separate the terminalia in order to eventually manipulate the genitalia at optimal observational angles. The terminalia were then individually macerated in a mixture of $2 \mathrm{~mL}$ of $10 \%(\mathrm{w} / \mathrm{v})$ potassium hydroxide $(\mathrm{KOH})$ solution and $0.12 \mathrm{~mL}$ of $95 \%$ ethanol in each well of 
a 12-well plate for $4 \mathrm{~d}$. Specimens were rinsed once with distilled water to remove the caustic $\mathrm{KOH}$ solution and transferred into a $0.85 \%$ sodium chloride $(\mathrm{NaCl})$ solution on a dissecting plate so that the genitalia could be dissected from the terminalia. Male genitalia and female ovipositors were gently separated from the macerated tissue using fine needles (No. 23) and sharp forceps under the stereomicroscope (Olympus). Each specimen was photographed using a digital camera (Samsung S700; Samsung, Seoul, Korea) and illustrated by painting with Chinese ink.

\subsection{Scanning Electron Microscopy}

Three other specimens of each sex were examined by scanning electron microscopy. All specimens were dissected as previously described in the light microscopic portion of this study. The terminalia were cleaned by soaking in phosphate buffer (PB, pH 7.4) to remove contamination artifacts. After that, they were primary fixed in $2.5 \%$ glutaraldehyde at $4{ }^{\circ} \mathrm{C}$ for $24 \mathrm{~h}$. They were then rinsed with PB three times at 15 min intervals and post-fixed with $1 \%$ osmium tetroxide at room temperature for $1 \mathrm{~h}$. Postfixation was followed by rinsing again as previously described and dehydrating with increasing alcohol concentrations as follow: $70 \%, 80 \%, 90 \%$, and $95 \%$. Specimens were left in each alcohol concentration for $12 \mathrm{~h}$. After the gradual dehydration process in the alcohol series, the specimens were dehydrated with absolute alcohol for two $12 \mathrm{~h}$ periods. Finally, they were subjected to critical point drying, attached to aluminum stubs with carbon double-stick tape, and coated with gold in a sputter-coating apparatus. The gold-coated specimens were then examined under a JEOL JSM-6610LV scanning electron microscope (JEOL, Peabody, MA, USA) at the Medical Science Research Equipment Center, Faculty of Medicine, Chiang Mai University in Chiang Mai, Thailand. The terminology used for most fly structures in this study follows the descriptions of Bernhard and Haenni [8], but the sensilla terminology specifically follows that of Zacharuk [9].

\section{Results}

A total of 36 specimens of $S$. discolor were examined by light and scanning electron microscopy. The average body length (head-abdomen) of all specimens was $6.88 \mathrm{~mm}$, varying from $4.80-9.30 \mathrm{~mm}$, but the distinct features were similar. Characteristic features of the terminalia of $S$. discolor are softly sclerized and formed posterior to the fifth abdominal segment as external reproductive organs. Examination under light microscopy showed that male terminalia were hardened and consisted of epandria, surstyli, and cerci (Figure 2A), whereas the tube-like ovipositor of the female consisted of the slightly sclerotized plates of sternites and tergites $6-8$, the supra-anal plate (SPAP), and the subanal plate (SBAP) coupled with the cerci (Figure 2C). Various kinds of microtrichia are present on the surface of the genitalia of both sexes. The extended aedeagus is illustrated in Figure 2B.

The dorsal view of the male genitalia under SEM revealed prominent features of the cercus, surstylus, and epandrium (Figure 3A). The cerci are slender and diverge distally with tapering tips (Figure $3 \mathrm{~A}$ ). The medial edges of the cerci are joined toward the base and are raised dorsally above the surface as a ridge (Figure 3A,B). The integument of the cercus is mostly covered in the proximal half with sensilla basiconica with short hair shafts and sparsely covered with sensilla trichodea (Figure 3B). The surstylus is basally enlarged in the proximal half and gradually tapered posteriorly in the distal half (Figure 3A). Strong bristles are only located in the proximal half of the surstylus. The medially located cerci are significantly longer and more slender than the surstyli that flank them laterally (Figure 3A). The epandria are densely covered with microtrichia and strong bristles (Figure 3A). SEM micrographs of the internal male genitalia or aedeagus revealed some more prominent components of the organ consisting of the pregonite, postgonite, phallic tube, juxta, and median stylus (Figure 3C). Surfaces of the postgonite, phallic tube, and juxta are all smooth, with the exception of a single, strong preapical bristle on the postgonite (Figure 3C). The pregonite bears numerous sensilla basiconica with short hair shafts as seen in Figure 3D. Within the juxta at the base of the aedeagus, the membranous tubular stylus is roughened on the surface and expanded apically (Figure 3E). Near the center of the extended aedeagus, a serrated median, dorsal process was presented (Figure 3F). 
The ovipositor of $S$. discolor is a flexible tubular structure which is usually withdrawn and concealed inside the abdomen. It extends outward from the fifth abdominal segment when depositing eggs. This structure is composed of more sclerotized plates consisting of paired tergites 6-8, sternites 6-8, paired cerci, the supra-anal plate (SPAP), and the subanal plate (SBAP), all joined together by a softer, less sclerotized membranous exoskeleton (Figure 2C). A dorsal view of the ovipositor under SEM showed that the sixth tergites are relatively smooth sclerotized plates with only a group of hairs at their apices (Figure 4A). In the ventral view under SEM (Figure 4C), sternites 6-8 are seen to be covered with numerous short hairs and a few long bristles near the apex of each. Sensilla trichoid are widespread on the external ovipositor and subanal plate (SBAP) (Figure 4C). The gonopore is located distally in the ovipositor (Figure 4B,C). Higher magnification provided by SEM reveals sensilla basiconica on the subanal plate (SBAP) as is pointed out in Figure 4D.
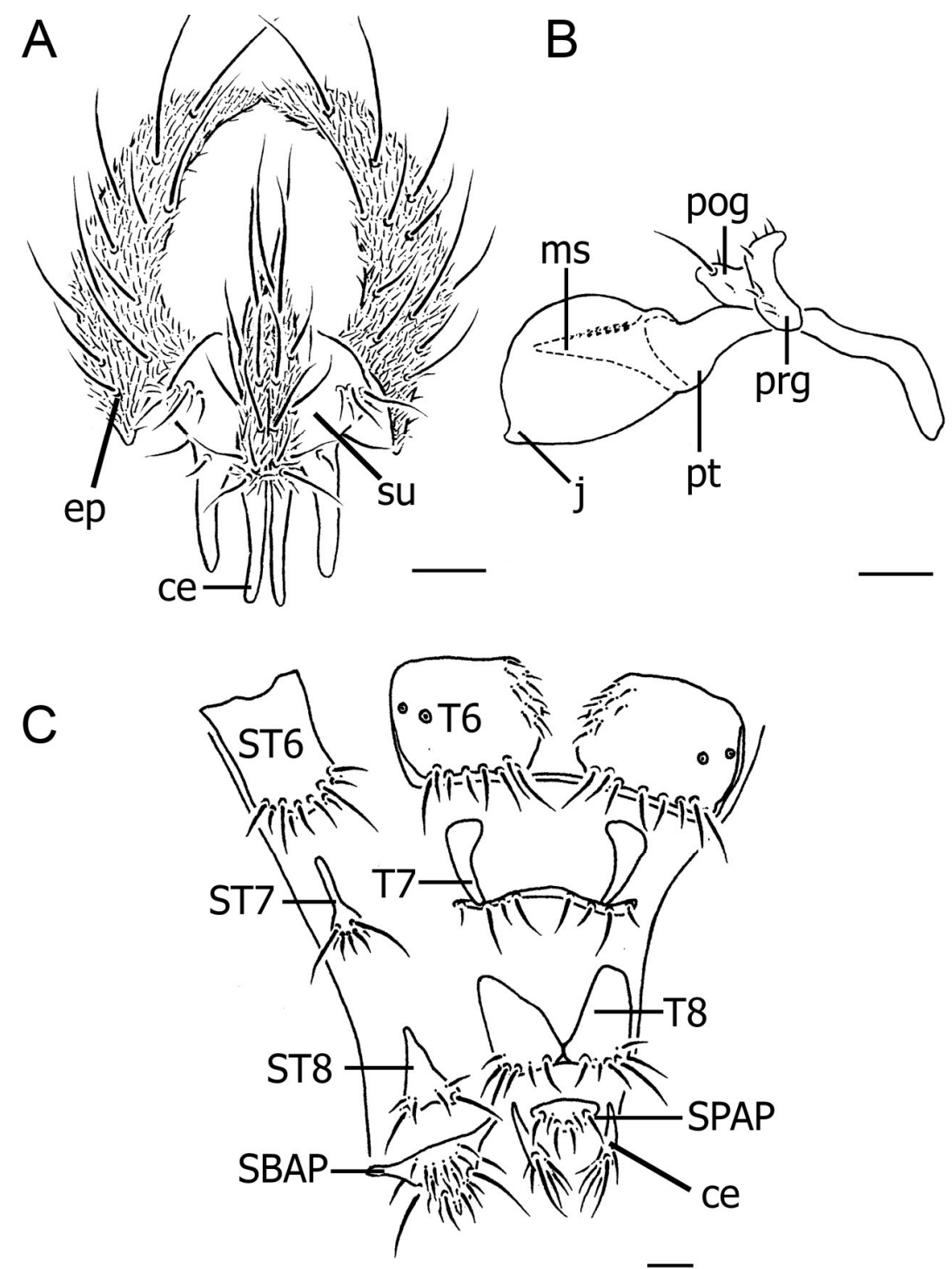

Figure 2. Illustrations of genitalia of Stomorhina discolor. (A) Posterior view of external male genitalia demonstrating prominent features: cercus (ce), surstylus (su), and epandrium (ep). Paired longitudinal cerci (ce) lie medial to the shorter surstyli (su) (bar = $2 \mathrm{~mm}$ ); (B) Lateral view of aedeagus showing pregonite (prg), postgonite (pog), phallic tube (pt), median stylus (ms), and juxta (j) (bar = $2 \mathrm{~mm}$ ); (C) Ovipositor showing tergite 6 (T6), sternite 6 (ST6), tergite 7 (T7), sternite 7 (ST7), tergite 8 (T8), sternite 8 (ST8), cercus (ce), subanal plate (SBAP), and supra-anal plate (SPAP) (bar = $0.1 \mathrm{~mm})$. 

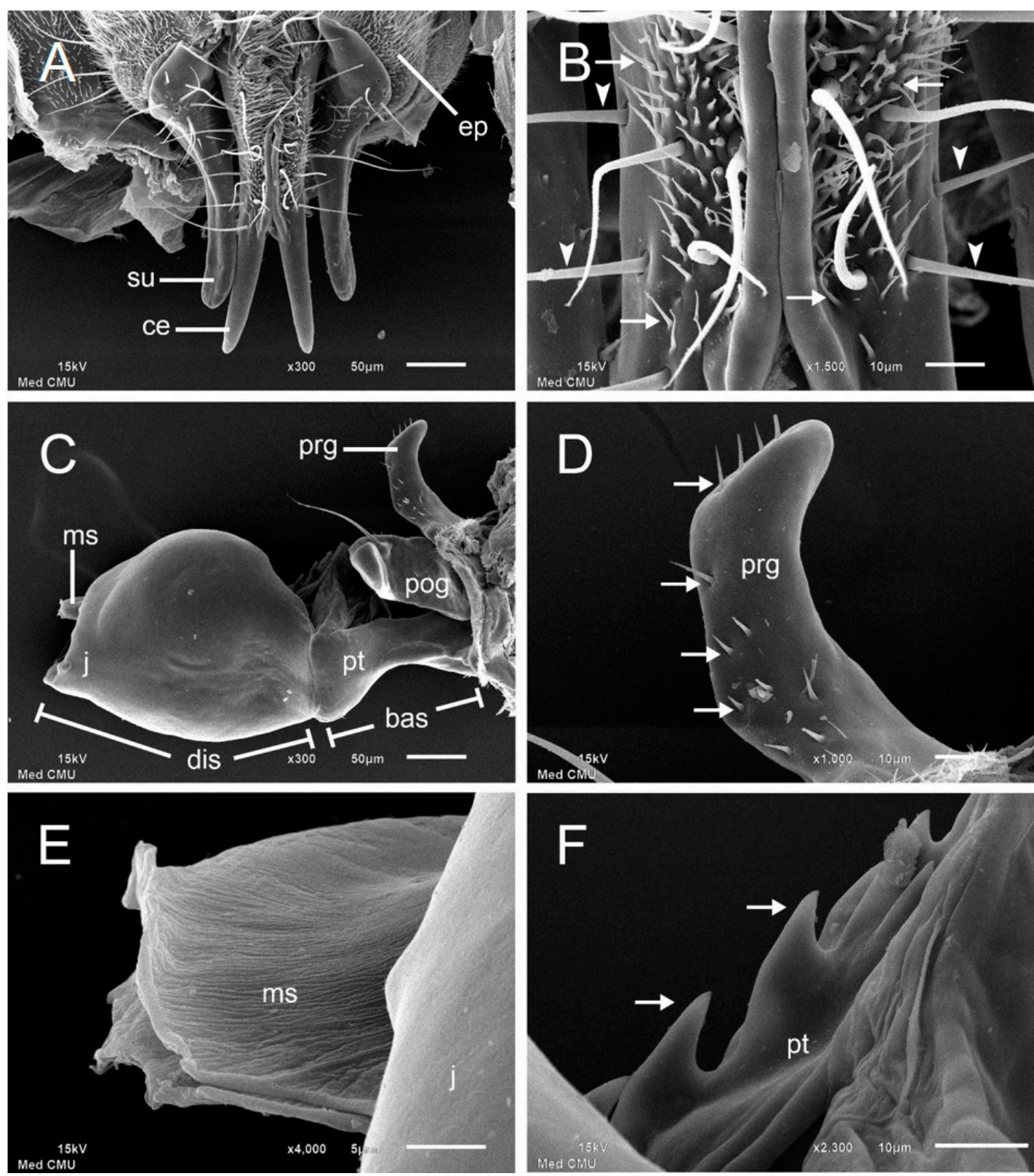

Figure 3. SEM micrographs of external male genitalia of Stomorhina discolor. (A) Posterior view of genitalia demonstrating prominent features: cercus (ce), surstylus (su), and epandrium (ep). Paired longitudinal cerci (ce) lie medial to the shorter surstyli (su); (B) High magnification of proximal portion of cerci showing sensilla trichodea of variable lengths (arrowheads) and sensilla basiconica with short hair shafts (arrows); (C) Lateral view of aedeagus showing pregonite (prg), postgonite (pog), phallic tube (pt), basiphallus (bas), distiphallus (dis), juxta (j), and median stylus (ms). The median stylus (ms) is inserted within the apically-oriented juxta (j). The pregonite (prg) and postgonite (pog) bearing a single bristle are prominent projections at the base; (D) High magnification of pregonite (prg) revealing sensilla basiconica with short hair shafts (arrows); (E) High magnification of median stylus (ms) with rough surface and expanded apex; (F) High magnification of phallic tube with dorsal serration (arrows) near center of aedeagus. 

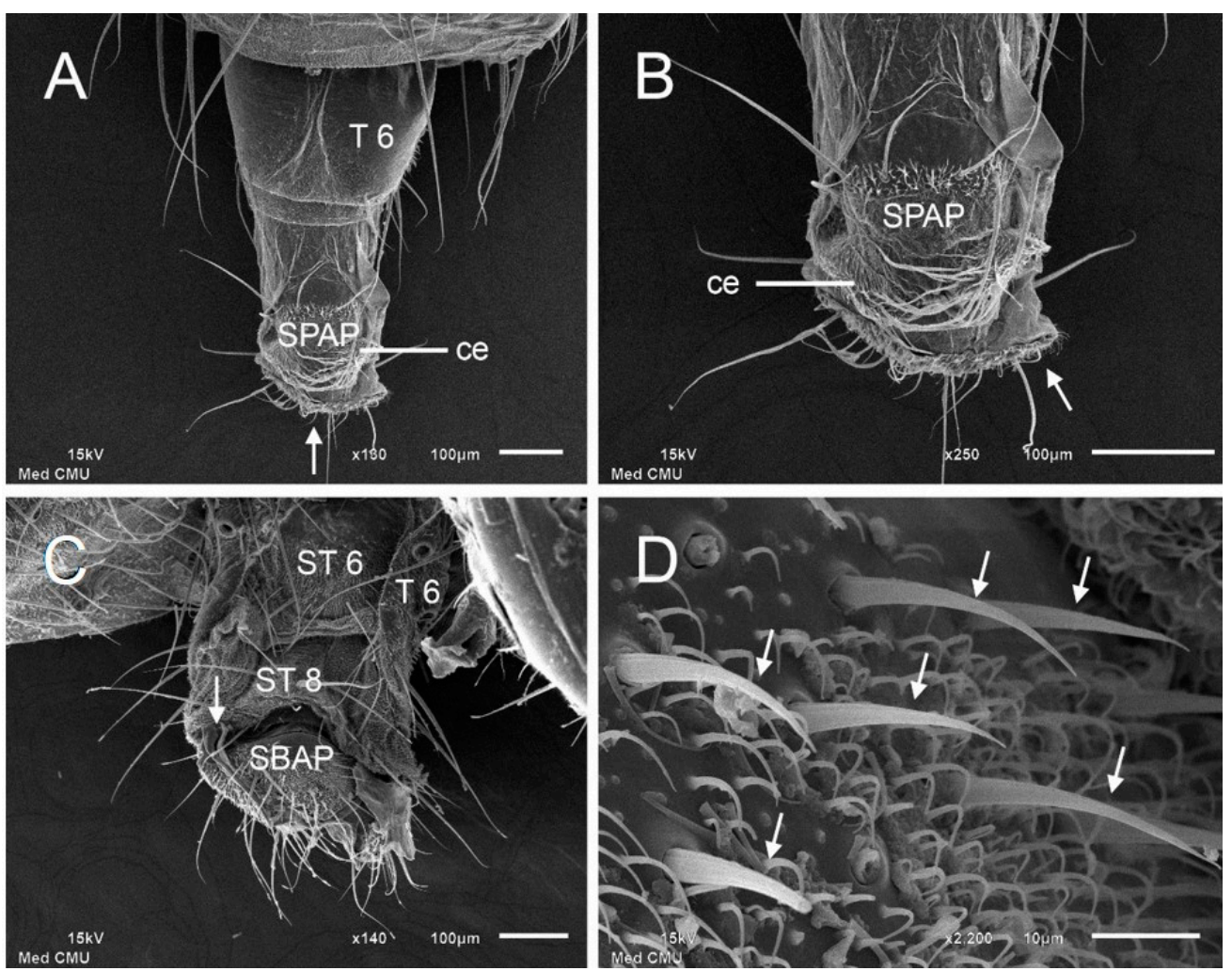

Figure 4. SEM micrographs of ovipositor of Stomorhina discolor. (A) Dorsal view of ovipositor showing tergite 6 (T6), supra-anal plate (SPAP), and cercus (ce). Gonopore at apex of ovipositor (arrow); (B) High magnification of dorsal view of ovipositor showing more detail of supra-anal plate (SPAP) and cercus (ce). Gonopore (arrow); (C) Ventral view of ovipositor showing side of tergite 6 (T6), sternite 6 (ST6), sternite 8 (ST8), and subanal plate (SBAP). Gonopore (arrow); (D) High magnification of subanal plate (SBAP) showing sensilla basiconica (arrows) and smaller microtrichia.

\section{Discussion}

A more complete description of the external genitalia of both sexes of $S$. discolor is being reported for the first time in this study. Distinct characteristics of the terminalia of $S$. discolor are unique to the species and are significantly different from those of other blow fly species. Illustrations of select male genitalia of some species of the genus Stomorhina of the British India fauna (including S. discolor) have been previously published [4]. Results of the current study have some similarity to the study of Senior-White et al. [4]; however, more specific details of each component of the genitalia are demonstrated in this study. Rognes [10] provided illustrations of the male and female genitalia of Stomorhina lunata (Fabricius) which are distinctly different from S. discolor (Table 1). Two types of sensilla, including sensilla trichodea and sensilla basiconica, were found on both the male and female genitalia of S. discolor in the present study. Likewise, the ovipositor of Ceylonomyia nigripes (Aubertin) is equipped with both sensilla trichodea and sensilla basiconica, in addition to sensilla placodea and sensilla styloconica [11]. Sensilla on terminalia are used for the positioning of genitalia for copulation during mating events [12]. Wang et al. [13] examined the expression pattern of an odorant receptor in the ovipositor of the blow fly Lucilia sericata (Meigen). Therefore, sensilla on the ovipositor may also have an olfactory function. In addition, sensilla on the female terminalia of the mosquito Aedes aegypti (Linnaeus) have been proposed to play a role in copulation as well as in ovipositional behavior [14]. However, sensilla of identical structures possibly serve different functions in different species of fruit fly, Drosophila spp. [12]. 
Table 1. Comparison male and female terminalia of Stomorhina discolor (current study) and Stomorhina lunata [10].

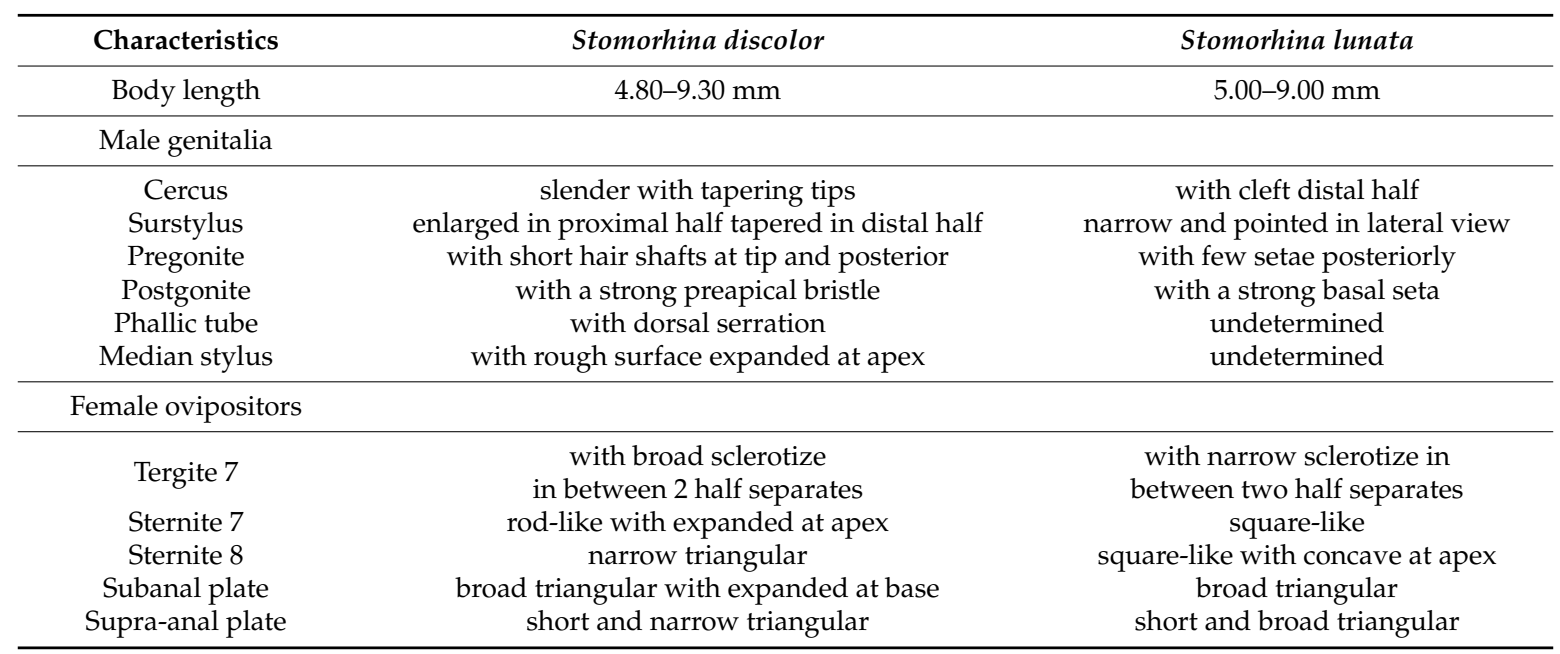

\section{Conclusions}

In conclusion, this study provides several distinctive details of the external terminalia of $S$. discolor which are not only useful for taxonomic purposes, but also provide important revelations that may be useful in future functional studies of the external genitalia of this fly species.

Acknowledgments: We are grateful for the financial support from the Naresuan University research fund (R2556C003 to Nophawan Bunchu). Staff of the Medical Science Research Equipment Center, Faculty of Medicine, Chiang Mai University in Chiang Mai, Thailand, are also acknowledged for technical support with the SEM.

Author Contributions: Kittikhun Moophayak performed experiments (collected the specimens, dissected the specimens for LM and SEM, and illustrated male genitalia and female ovipositor), prepared the manuscript and figures. Sangob Sanit performed experiments (prepared the specimens for SEM), prepared all figures. Hiromu Kurahashi, Kom Sukontason, Tarinee Chaiwong analyzed the data, prepared the manuscript. Kabkaew L. Sukontason, Roy C. Vogtsberger performed experiments (SEM), edited the manuscript. Nophawan Bunchu designed the experiments, performed the experiments (identified the specimens), and finally approved the manuscript. All authors read and approved the final manuscript.

Conflicts of Interest: The authors declare no conflict of interest.

\section{References}

1. Dear, J.P. A revision of Australian Rhiniinae (Diptera: Calliphoridae). Aust. J. Zool. 1977, 25, $779-826$. [CrossRef]

2. Kurahashi, H.; Bunchu, N. The blow flies recorded from Thailand, with the description of a new species of Isomyia Walker (Diptera: Calliphoridae). Jpn. J. Syst. Entomol. 2011, 17, 237-278.

3. Kurahashi, H.; Leh, M.U. The blow flies from Sarawak, East Malaysia (Diptera: Calliphoridae), with practical keys and a checklist. Sarawak Mus. J. 2009, 87, 299-300.

4. Senior-White, R.; Aubertin, D.; Smart, J. Diptera: Family Calliphoridae. In The Fauna of British India, Including the Remainder of the Oriental Region; Sewell, R.B.S., Ed.; Taylor \& Francis: London, UK, 1940.

5. Verves, Y.G. A catalogue of Oriental Calliphoridae (Diptera). Int. J. Res. 2005, 16, 233-310.

6. Bunchu, N. Blow fly (Diptera: Calliphoridae) in Thailand: Distribution, morphological identification and medical importance appraisals. Int. J. Parasitol. Res. 2012, 4, 57-64. [CrossRef]

7. Kutty, S.N.; Pape, T.; Wiegmann, B.M.; Meier, R. Molecular phylogeny of the Calyptrate (Diptera: Cyclorrhapha) with an emphasis on the superfamily Oestroidea and the position of Mystacinobiidae and McAlpine's fly. Syst. Entomol. 2010, 35, 614-635. [CrossRef] 
8. Bernhard, M.; Haenni, J.P. Morphology and terminology of adult Diptera (other than terminalia). In Contributions to a Manual of Palaearctic Diptera. General and Applied Dipterology; László, P., Béla, D., Eds.; Science Herald: Budapest, Hungary, 2000; pp. 22-51.

9. Zacharuk, R.Y. Antennal sensilla. In Comparative Insect Physiology, Biochemistry and Pharmacology; Kerkut, G.A., Gilbert, L.I., Eds.; Pergamon Press: Oxford, NY, USA, 1985; pp. 1-69.

10. Rognes, K. Blowflies (Diptera, Calliphoridae) of Fennoscandia and Denmark. In Fauna Entomologica Scandinavica; Scandinavian Science Press Ltd.: Leiden, The Netherlands, 1991.

11. Ngern-Klun, R.; Sukontason, K.; Methanitikorn, R.; Vogtsberger, R.C.; Sukontason, K.L. Fine structure of Chrysomya nigripes (Diptera: Calliphoridae), a fly species of medical importance. Parasitol. Res. 2007, 100, 993-1002. [CrossRef] [PubMed]

12. Acebes, A.; Cobb, M.; Feurler, J.F. Species-Specific effects of single sensillum ablation on mating position in Drosophila. J. Exp. Biol. 2003, 206, 3095-3100. [CrossRef] [PubMed]

13. Wang, X.; Zhong, M.; Wen, J.; Cai, J.; Jiang, H.; Liu, Y.; Aly, S.; Xiong, F. Molecular characterization and expression pattern of an odorant receptor from the myiasis-causing blowfly, Lucilia sericata (Diptera: Calliphoridae). Parasitol. Res. 2003, 110, 843-851. [CrossRef] [PubMed]

14. Rossignol, P.A.; McIver, S.B. Fine structure and role in behavior of sensilla on the terminalia of Aedes aegypti (L.) (Diptera: Culicidae). J. Morphol. 1977, 151, 419-437. [CrossRef] [PubMed]

(C) 2017 by the authors; licensee MDPI, Basel, Switzerland. This article is an open access article distributed under the terms and conditions of the Creative Commons Attribution (CC-BY) license (http:/ / creativecommons.org/licenses/by/4.0/). 